\title{
On quantum conditional entropies defined in terms of the $f$-divergences
}

\author{
Alexey E. Rastegin \\ Department of Theoretical Physics, Irkutsk State University, Gagarin Bv. 20, Irkutsk 664003, Russia
}

\begin{abstract}
We consider a family of quantum conditional entropies based on the concept of quantum $f$ divergences. First, we explicitly formulate conditions under which the notion of quantum conditional entropy is well defined in this way. In particular, we demand that the value of conditional entropy be independent of any extension of the principal Hilbert space. Using fundamental properties of the quantum $f$-divergences, several interesting relations for such conditional entropies are formulated. We separately examine an especially important case of quantum conditional entropies related to the Tsallis divergence.
\end{abstract}

Keywords: $f$-divergence, monotonicity, quantum conditional entropy

\section{INTRODUCTION}

The concept of relative entropy is of great importance in information theory. Many principal results in quantum information theory are closely related to properties of the relative entropy. Its monotonicity and joint convexity are very essential [1, 2]. The Shannon entropy of probability distributions and the von Neumann entropy of density matrices are commonly used measures of an informational content. Other entropic functions have found to be useful in various questions [3]. There exist reasons to study possible extensions of the standard divergence. Many of them can be unified within the concept of $f$-divergences [4]. This approach is a quantum counterpart of Csiszár's $f$-divergences [5]. Petz's quasi-entropies are a primary example [6]. Quantum divergences may be adopted as distance measures, though they are not a metric in the formal sense. In studying problems of information theory, a collection of distinguishability measures is typically used [7]. For example, the authors of [8] recently proposed one-shot generalizations for usual mutual information related to the von Neumann entropy.

The conditional entropy is widely used in information theory [9]. In the classical regime, the conditional Rényi [10] and Tsallis [11] entropies are examples of generalized conditional entropies. The Rényi entropy [12] and the Tsallis entropy [13] are well known extensions of the Shannon entropy. Relations between generalized conditional entropies and the error probability are of interest in communication [14]. In the quantum regime, the notion of conditional entropy is also very important [15, 16]. There are more than one way to fit an approach to quantum conditional entropies. The quantum conditional entropy is usually written as the difference of corresponding von Neumann entropies [17]. It is closely related to the strong subadditivity [18]. The conditional entropy can also be expressed in terms of the standard relative entropy [2, 19]. An approach based on relevant divergences was developed in details for some interesting cases [20 22]. To study entanglement in a bipartite system, the authors of [23] proposed a modification of the conditional entropy after a local measurement on one of the subsystems.

The aim of the present paper is to study quantum conditional entropies based on the notion of $f$-divergences. The paper is organized as follows. In Sect. II the preliminary material is given. In particular, we discuss required properties of the quantum $f$-divergences. Corresponding conditional entropies are defined in Sect. [II] We consider general conditions, when the presented extension is well defined. Particularly, we demand that the value of conditional entropy be independent of any extension of the state space. When the function used in the $f$-divergence enjoys certain conditions, the considered entropic quantities obey some interesting relations. These results are generally discussed in Sect. IV] In Sect. V] we separately analyze the case of Tsallis entropies. Some additional properties of the conditional $\alpha$-entropies of Tsallis type are derived. In Sect. VI] we conclude the paper with a summary of results.

\section{PRELIMINARIES}

In this paper, we will study quantum conditional entropies defined on base of the quantum $f$-divergences. The latter is a quantum counterpart of the Csiszár $f$-divergence [5]. This concept provide a unified approach to studying relative entropies in the classical regime. Let $\xi \mapsto f(\xi)$ be a convex function on $\xi \in[0 ;+\infty)$ with $f(1)=0$. The Csiszár $f$-divergence of the probability distribution $\left\{p_{x}\right\}$ from $\left\{q_{x}\right\}$ is defined as [5]

$$
S_{f}(p \| q):=\sum_{x} q_{x} f\left(\frac{p_{x}}{q_{x}}\right) .
$$


Using the Jensen inequality for a convex function, we easily obtain

$$
\sum_{x} q_{x} f\left(\frac{p_{x}}{q_{x}}\right) \geq f\left(\sum_{x} p_{x}\right)=f(1)
$$

or merely $S_{f}(p \| q) \geq 0$. In other words, the $f$-divergence takes nonnegative values, and $S_{f}(p \| p)=0$. Several approaches to defining divergences and other entropic measures are reviewed in [24]. Without explicit assignment of the function, various inequalities with the Csiszár $f$-divergences were obtained in [25].

Let $\mathcal{H}$ be a finite-dimensional Hilbert space. By $\mathcal{L}(\mathcal{H})$ and $\mathcal{L}_{+}(\mathcal{H})$, we denote the space of linear operators on $\mathcal{H}$ and the set of positive semi-definite ones. Eigenvalues of an operator $X \in \mathcal{L}(\mathcal{H})$ form its $\operatorname{spectrum} \operatorname{spec}(X)$. $B y \operatorname{ran}(X)$, we mean the range of operator $X$. For positive $A$, the subspace $\operatorname{ran}(A)$ is spanned by those eigenvectors of $A$ that correspond to strictly positive eigenvalues. For $\mathrm{X}, \mathrm{Y} \in \mathcal{L}(\mathcal{H})$, we define the Hilbert-Schmidt inner product

$$
\langle\mathrm{X}, \mathrm{Y}\rangle_{\mathrm{hs}}:=\operatorname{tr}\left(\mathrm{X}^{\dagger} \mathrm{Y}\right)
$$

In the following, we use the convention that powers of a positive operator are taken only on its support. For any $\mathrm{A} \in \mathcal{L}_{+}(\mathcal{H})$, by $\mathrm{A}^{0}$ we mean the orthogonal projector onto $\operatorname{ran}(\mathrm{A})$. In the finite-dimensional case, we further define $P \vee Q$ as the projector onto the sum of subspaces $\operatorname{ran}(P)+\operatorname{ran}(Q)$. The last definition should be modified in the infinite-dimensional case, since the sum of two closed subspaces is not necessarily closed. In the following, we restrict a consideration to finite dimensions.

Any state of a quantum system is described by density operator $\boldsymbol{\rho} \in \mathcal{L}_{+}(\mathcal{H})$. We usually deal with normalized states such that $\operatorname{tr}(\boldsymbol{\rho})=1$. By $\varrho_{*}=d^{-1} \mathbb{1}$ with the identity operator $\mathbb{1}$, we mean the completely mixed state in $d$ dimensions. For density operators $\boldsymbol{\rho}$ and $\boldsymbol{\sigma}$, the quantum relative entropy is expressed as [2]

$$
D_{1}(\boldsymbol{\rho} \| \boldsymbol{\sigma}):= \begin{cases}\operatorname{tr}(\boldsymbol{\rho} \ln \boldsymbol{\rho}-\boldsymbol{\rho} \ln \boldsymbol{\sigma}), & \text { if } \operatorname{ran}(\boldsymbol{\rho}) \subseteq \operatorname{ran}(\boldsymbol{\sigma}) \\ +\infty, & \text { otherwise }\end{cases}
$$

There exist several generalizations of the quantity (2.4). Many of them can be described as partial cases on the quantum $f$-divergence [4]. Taking $\mathrm{A}, \mathrm{B} \in \mathcal{L}_{+}(\mathcal{H})$, we introduce the left multiplication $\Lambda_{\mathrm{A}}$ and the right multiplication $\Upsilon_{\mathrm{B}}$, namely

$$
\Lambda_{\mathrm{A}}: \mathrm{X} \mapsto \mathrm{AX}, \quad \Upsilon_{\mathrm{B}}: \mathrm{X} \mapsto \mathrm{XB}
$$

where $X \in \mathcal{L}(\mathcal{H})$. Left and right multiplications commute with each other, i.e., $\Lambda_{\mathrm{A}} \Upsilon_{\mathrm{B}}=\Upsilon_{\mathrm{B}} \Lambda_{\mathrm{A}}$ for $\mathrm{A}, \mathrm{B} \in \mathcal{L}_{+}(\mathcal{H})$. In terms of the corresponding projectors, we write the spectral decompositions

$$
\mathrm{A}=\sum_{a \in \operatorname{spec}(\mathrm{A})} a \mathrm{P}_{a}, \quad \mathrm{~B}=\sum_{b \in \operatorname{spec}(\mathrm{B})} b \mathrm{Q}_{b} .
$$

Let $\xi \mapsto f(\xi)$ be a real-valued function on $\xi \in[0 ;+\infty)$ such that it is continuous on $(0,+\infty)$ and the limit

$$
\ell(f):=\lim _{\xi \rightarrow+\infty} \xi^{-1} f(\xi)
$$

exists in $[-\infty ;+\infty]$. Using the set $\left\{a b^{-1}: a \in \operatorname{spec}(\mathrm{A}), b \in \operatorname{spec}(\mathrm{B})\right\}$, we write [4]

$$
f\left(\Lambda_{\mathrm{A}} \Upsilon_{\mathrm{B}^{-1}}\right):=\sum_{a \in \operatorname{spec}(\mathrm{A})} \sum_{b \in \operatorname{spec}(\mathrm{B})} f\left(a b^{-1}\right) \Lambda_{\mathrm{P}_{a}} \Upsilon_{\mathrm{Q}_{b}} .
$$

In the right-hand side of (2.8), we assume that $\operatorname{ran}(\mathrm{A}) \subseteq \operatorname{ran}(\mathrm{B})$. Then the $f$-divergence of $A$ with respect to $B$ is defined by [4]

$$
D_{f}(\mathrm{~A} \| \mathrm{B}):=\left\langle\mathrm{B}^{1 / 2}, f\left(\Lambda_{\mathrm{A}} \Upsilon_{\mathrm{B}^{-1}}\right) \mathrm{B}^{1 / 2}\right\rangle_{\mathrm{hs}}
$$

When $\operatorname{ran}(\mathrm{A}) \nsubseteq \operatorname{ran}(\mathrm{B})$, the quantum $f$-divergence is defined by the formula [4]

$$
D_{f}(\mathrm{~A} \| \mathrm{B}):=\lim _{\varepsilon \searrow 0} D_{f}(\mathrm{~A} \| \mathrm{B}+\varepsilon \mathbb{1}) .
$$

Taking $f_{1}(\xi)=\xi \ln \xi$, we obtain the standard relative entropy (2.4) rewritten for positive operators of any trace. Namely, for $\operatorname{ran}(A) \subseteq \operatorname{ran}(B)$ we have

$$
D_{1}(\mathrm{~A} \| \mathrm{B}):=\operatorname{tr}(\mathrm{A} \ln \mathrm{A}-\mathrm{A} \ln \mathrm{B})
$$


Basic properties of the $f$-divergence (2.10) are examined in [4]. Under the above conditions on the function $\xi \mapsto f(\xi)$, the $f$-divergence is continuous in its second entry [4]. It must be stressed that continuity in the first entry does not hold in general [4].

Similarly to (2.2), we can check positivity of the $f$-divergence in some important cases. Consider two normalized states

$$
\boldsymbol{\rho}=\sum_{a \in \operatorname{spec}(\boldsymbol{\rho})} a \boldsymbol{\pi}_{a}, \quad \boldsymbol{\sigma}=\sum_{b \in \operatorname{spec}(\boldsymbol{\sigma})} b \boldsymbol{\lambda}_{b} .
$$

In the case $\operatorname{ran}(\boldsymbol{\rho}) \subseteq \operatorname{ran}(\boldsymbol{\sigma})$, the divergence (2.9) can be represented as

$$
D_{f}(\boldsymbol{\rho} \| \boldsymbol{\sigma})=\sum_{a \in \operatorname{spec}(\boldsymbol{\rho})} \sum_{b \in \operatorname{spec}(\boldsymbol{\sigma}) \backslash\{0\}} b f\left(\frac{a}{b}\right) \operatorname{tr}\left(\boldsymbol{\pi}_{a} \boldsymbol{\lambda}_{b}\right) .
$$

This expression directly follows from item 2.3 of [4]. The factors $b \operatorname{tr}\left(\boldsymbol{\pi}_{a} \boldsymbol{\lambda}_{b}\right)$ in the sum (2.13) can be treated as probabilities due to the relation

$$
\sum_{a \in \operatorname{spec}(\boldsymbol{\rho})} \sum_{b \in \operatorname{spec}(\boldsymbol{\sigma}) \backslash\{0\}} b \operatorname{tr}\left(\boldsymbol{\pi}_{a} \boldsymbol{\lambda}_{b}\right)=\operatorname{tr}(\boldsymbol{\sigma})=1 .
$$

Because of $\operatorname{ran}(\boldsymbol{\rho}) \subseteq \operatorname{ran}(\boldsymbol{\sigma})$, we further have

$$
\sum_{a \in \operatorname{spec}(\boldsymbol{\rho})} \sum_{b \in \operatorname{spec}(\boldsymbol{\sigma}) \backslash\{0\}} a \operatorname{tr}\left(\boldsymbol{\pi}_{a} \boldsymbol{\lambda}_{b}\right)=\operatorname{tr}(\boldsymbol{\rho})=1 .
$$

Combining these facts with (2.13) and convexity of the function $\xi \mapsto f(\xi)$ finally gives

$$
D_{f}(\boldsymbol{\rho} \| \boldsymbol{\sigma}) \geq f(1),
$$

whence $D_{f}(\boldsymbol{\rho} \| \boldsymbol{\sigma}) \geq 0$ in the case $f(1) \geq 0$. The result (2.16) is a quantum counterpart of (2.2). The $f$-divergence of normalized $\boldsymbol{\rho}$ with respect to normalized $\boldsymbol{\sigma}$ is nonnegative, when $\operatorname{ran}(\boldsymbol{\rho}) \subseteq \operatorname{ran}(\boldsymbol{\sigma})$ and the used convex function obeys $f(1) \geq 0$. This conclusion can be extended to any two operators $\mathrm{A}, \mathrm{B} \in \mathcal{L}_{+}(\mathcal{H})$ such that $\operatorname{ran}(\mathrm{A}) \subseteq \operatorname{ran}(\mathrm{B})$ and $\operatorname{tr}(\mathrm{A})=\operatorname{tr}(\mathrm{B})$. Here, we merely use the homogeneity [4]

$$
D_{f}(\lambda \mathrm{A} \| \lambda \mathrm{B})=\lambda D_{f}(\mathrm{~A} \| \mathrm{B})
$$

where $\lambda \in[0 ;+\infty)$. Approaching quantum conditional entropies on base of the $f$-divergences, we will use operators with unequal traces. In this case, corresponding divergences may take negative values.

Some existing extensions of the relative entropy (2.4) do not directly follow from the definition (2.9). The Rényi divergence is most important of them. In some respects, the traditional form of quantum Rényi's divergence is not satisfactory. Recently, Müller-Lennert et al. 22] and Wilde et al. 26] proposed a new definition of quantum Rényi's divergence. Further extensions of this approach are considered in [27]. The writers of [26] used the "sandwiched" relative Rényi entropy in studying a strong converse for the classical capacity of entanglement-breaking and Hadamard channels. For the pure-loss bosonic channel, this issue is examined in [28]. Some desired properties of the new definition were shown in a limited range of parameters and conjectured for a larger range. The conjectures have been proved soon in [29, 30]. The authors of [31] compared the old and new forms of Rényi's $\alpha$-divergences in the context of quantum hypothesis testing. Hence, the proper choice seems to be the traditional definition for $\alpha<1$ and the new definition for $\alpha>1$ [31]. In the following, we will focus an attention on the family of quantum $f$-divergences defined by (2.9).

Many results of quantum information theory are related to monotonicity of the standard relative entropy [1, 19]. The most general form of quantum evolution is described by completely positive maps [19]. Consider a linear map $\Phi: \mathcal{L}(\mathcal{H}) \rightarrow \mathcal{L}\left(\mathcal{H}^{\prime}\right)$ that takes elements of $\mathcal{L}(\mathcal{H})$ to elements of $\mathcal{L}\left(\mathcal{H}^{\prime}\right)$. We call a map positive, if it maps each positive operator to positive one again. Let id" be the identity map on $\mathcal{L}\left(\mathcal{H}^{\prime \prime}\right)$, where the space $\mathcal{H}^{\prime \prime}$ is assigned to a reference system. The complete positivity implies that the map $\Phi \otimes \mathrm{id}^{\prime \prime}$ is positive for any dimension of $\mathcal{H}^{\prime \prime}$. A completely positive map $\Phi$ can be written as

$$
\Phi(\mathrm{X})=\sum_{n} \mathrm{~K}_{n} \mathrm{X} \mathrm{K}_{n}^{\dagger}
$$

Here, the Kraus operators $\mathrm{K}_{n}$ map the input space $\mathcal{H}$ to the output space $\mathcal{H}^{\prime}$. When physical process is closed and the probability is conserved, the map preserves the trace. Trace-preserving completely positive maps (TPCP-maps) 
are called quantum channels [3, 19]. Entropic characteristics of quantum channels were examined in [32, 33]. In some respects, this treatment can be extended with generalized entropic forms [34, 35].

Conditions for monotonicity of the quantum $f$-divergence are obtained in [4]. Let $\Phi$ be a TPCP-map. If the function $\xi \mapsto f(\xi)$ is operator convex on $[0 ;+\infty)$ then [4]

$$
D_{f}(\Phi(\mathrm{A}) \| \Phi(\mathrm{B})) \leq D_{f}(\mathrm{~A} \| \mathrm{B}) .
$$

The inequality (2.19) expresses monotonicity of the quantum $f$-divergences. It will be very important in studying quantum conditional entropies defined on base of the $f$-divergences.

\section{QUANTUM CONDITIONAL ENTROPIES}

Conditional entropies are used in considering multi-partite quantum systems. Let the state of a bipartite system be described by density matrix $\rho_{A B} \in \mathcal{L}_{+}\left(\mathcal{H}_{A B}\right)$, where $\mathcal{H}_{A B}=\mathcal{H}_{A} \otimes \mathcal{H}_{B}$. The conditional von Neumann entropy is defined as

$$
H_{1}\left(\boldsymbol{\rho}_{A B} \mid B\right):=H_{1}\left(\boldsymbol{\rho}_{A B}\right)-H_{1}\left(\boldsymbol{\rho}_{B}\right) .
$$

Here, the quantity $H_{1}(\boldsymbol{\rho})=-\operatorname{tr}(\boldsymbol{\rho} \ln \boldsymbol{\rho})$ is the von Neumann entropy of $\boldsymbol{\rho}$, and the reduced density matrix $\boldsymbol{\rho}_{B}$ is obtained from $\rho_{A B}$ by tracing-out the space $\mathcal{H}_{A}$. Some useful relations between operators before and after partial trace with applications to quantum entropies were obtained in [36]. Continuity of the conditional entropy (3.1) was considered in [37]. The definition (3.1) has a lot of consequences [19]. Here, we recall two properties, since they will be referred to in the following. We have [19]

$$
\begin{aligned}
& H_{1}\left(|A B\rangle\langle A B|| B)=-H_{1}\left(\boldsymbol{\rho}_{B}\right),\right. \\
& H_{1}\left(\boldsymbol{\rho}_{A} \otimes \boldsymbol{\rho}_{B} \mid B\right)=H_{1}\left(\boldsymbol{\rho}_{A}\right) .
\end{aligned}
$$

Thus, the conditional entropy (3.1) reduces to minus the entropy of $\boldsymbol{\rho}_{B}$ in the case of pure states $|A B\rangle \in \mathcal{H}_{A B}$. It is herewith strictly negative for pure entangled states. On the other hand, the conditional entropy (3.1) is nonnegative in the case of separable mixed states. Such properties support a treatment of the quantity (3.1) as a quantum counterpart of the classical conditional entropy.

Let us proceed to more general forms of quantum conditional information. One of possible ways is a direct extension of the definition (3.1) with generalized entropies. It turned out that an appropriate approach to obtaining more conditional entropies can be based on quantum divergences. The key observation is that the right-hand side of (3.1) can be rewritten in an alternative form [20, 21]:

$$
H_{1}\left(\boldsymbol{\rho}_{A B} \mid B\right):=-\inf \left\{D_{1}\left(\boldsymbol{\rho}_{A B} \| \mathbb{1}_{A} \otimes \boldsymbol{\sigma}_{B}\right): \boldsymbol{\sigma}_{B} \in \mathcal{L}_{+}\left(\mathcal{H}_{B}\right), \operatorname{tr}\left(\boldsymbol{\sigma}_{B}\right)=1\right\} .
$$

This way to obtain quantum conditional entropies has been realized for the so-called min-entropy and max-entropy in [20, 21]. With minor modifications, this approach was recently considered in the Rényi case [22].

Following the idea of [20, 38], the following definition will be used. For the given $f$-divergence, we define the associated quantum conditional entropy

$$
H_{f}\left(\boldsymbol{\rho}_{A B} \mid B\right):=-\inf \left\{D_{f}\left(\boldsymbol{\rho}_{A B} \| \mathbb{1}_{A} \otimes \boldsymbol{\sigma}_{B}\right): \boldsymbol{\sigma}_{B} \in \mathcal{L}_{+}\left(\mathcal{H}_{B}\right), \operatorname{tr}\left(\boldsymbol{\sigma}_{B}\right) \leq 1\right\} .
$$

As was mentioned above, the $f$-divergence is continuous in its second entry (for details, see section 2 of [4]). We also note that the optimization in (3.5) is taken over a compact set. According to Weierstrass' theorem, the infimum is finite and reached for at least one element of the set.

The optimization in (3.5) is taken over the set of all sub-normalized states. There exist several reasons to allow such states. For the min-entropy an optimization over the set of sub-normalized states is equivalent to an optimization over normalized states [38]. In this case, the optimization problem is linear [21]. Hence, convenient properties of the min-entropy follow. The given quantum system can always be imagined as a part of some larger quantum systems. It is natural to demand that the actual value of conditional entropies be independent of any extension of the state space. The following argument demonstrates the physical meaning behind the sub-normalized states in (3.5).

Under conditions imposed on the function $\xi \mapsto f(\xi)$ (see (2.7) and comments therein), the following property holds. If the four positive semi-definite operators $A_{1}, B_{1}, A_{2}, B_{2}$ obey $A_{1}^{0} \vee B_{1}^{0} \perp A_{2}^{0} \vee B_{2}^{0}$ then [4]

$$
D_{f}\left(\mathrm{~A}_{1}+\mathrm{A}_{2} \| \mathrm{B}_{1}+\mathrm{B}_{2}\right)=D_{f}\left(\mathrm{~A}_{1} \| \mathrm{B}_{1}\right)+D_{f}\left(\mathrm{~A}_{2} \| \mathrm{B}_{2}\right) .
$$


For arbitrary density operator $\boldsymbol{\rho}_{A B}$, we have (see lemma B.4.1 in [20])

$$
\operatorname{ran}\left(\boldsymbol{\rho}_{A B}\right) \subseteq \operatorname{ran}\left(\boldsymbol{\rho}_{A}\right) \otimes \operatorname{ran}\left(\boldsymbol{\rho}_{B}\right),
$$

where $\boldsymbol{\rho}_{A}=\operatorname{tr}_{B}\left(\boldsymbol{\rho}_{A B}\right)$ and $\boldsymbol{\rho}_{B}=\operatorname{tr}_{A}\left(\boldsymbol{\rho}_{A B}\right)$ are the reduced density matrices. Let two real numbers $\mu, \bar{\mu} \in[0 ; 1]$ satisfy $\mu+\bar{\mu} \leq 1$. Let the density matrices $\boldsymbol{\omega}_{B}$ and $\varpi_{B}$ be such that $\operatorname{tr}\left(\boldsymbol{\omega}_{B}\right)=\operatorname{tr}\left(\varpi_{B}\right)=1$, $\boldsymbol{\omega}_{B} \in \mathcal{L}_{+}\left(\operatorname{ran}\left(\boldsymbol{\rho}_{B}\right)\right)$, and $\varpi_{B}^{0} \perp \boldsymbol{\rho}_{B}^{0}$. We consider sub-normalized states of the form

$$
\boldsymbol{\sigma}_{B}^{\prime}=\mu \boldsymbol{\omega}_{B}+\bar{\mu} \varpi_{B}
$$

Using the property (3.6), for the case $f(0)=0$ one gets

$$
D_{f}\left(\boldsymbol{\rho}_{A B} \| \mathbb{1}_{A} \otimes \boldsymbol{\sigma}_{B}^{\prime}\right)=D_{f}\left(\boldsymbol{\rho}_{A B} \| \mathbb{1}_{A} \otimes \mu \boldsymbol{\omega}_{B}\right),
$$

since $D_{f}\left(\mathrm{~A}_{2} \| \mathrm{B}_{2}\right)$ is zero for $\mathrm{A}_{2}=\mathbf{0}$. In the right-hand side of (3.9), the state $\mu \boldsymbol{\omega}_{B}$ has the trace $\mu$. Except for $\bar{\mu}=0$, this state is strictly sub-normalized. This reason shows that sub-normalized states naturally occur in approaching the notion of conditional entropy in terms of quantum divergences.

We demand that the actual value of quantum conditional entropies be independent of a choice of considered Hilbert space. Of course, our consideration should involve at least the subspace $\operatorname{ran}\left(\boldsymbol{\rho}_{B}\right)$ of $\mathcal{H}_{B}$. This point is formally posed as follows. Let us treat the system $B$ as a particular case of extended quantum system $\widetilde{B}$ with the Hilbert space $\mathcal{H}_{\widetilde{B}}=\mathcal{H}_{B} \oplus \mathcal{K}$. For all density matrices $\boldsymbol{\rho}_{A B}$ on $\mathcal{H}_{A B}$ and arbitrary finite space $\mathcal{K}$, we formulate the condition

$$
H_{f}\left(\boldsymbol{\rho}_{A B} \mid B\right)=H_{f}\left(\boldsymbol{\rho}_{A B} \mid \widetilde{B}\right)
$$

It turns out that the claim (3.10) can be provided by imposing some conditions on the used function $f$. We have the following statement.

Theorem 1 Let twice continuously differentiable function $\xi \mapsto f(\xi)$ be operator convex on $[0 ;+\infty)$, let the limit 2.7$)$ exist in $[-\infty ;+\infty]$, and let $f(0)=0$. Then the quantum conditional entropy (3.5) is represented as

$$
H_{f}\left(\boldsymbol{\rho}_{A B} \mid B\right)=-\inf \left\{D_{f}\left(\boldsymbol{\rho}_{A B} \| \mathbb{1}_{A} \otimes \boldsymbol{\sigma}_{B}\right): \boldsymbol{\sigma}_{B} \in \mathcal{L}_{+}\left(\operatorname{ran}\left(\boldsymbol{\rho}_{B}\right)\right), \operatorname{tr}\left(\boldsymbol{\sigma}_{B}\right)=1\right\} .
$$

Proof. We aim to show that the definition (3.5) is actually reduced to (3.11). We first note that the optimization in (3.5) can be taken over states of the form (3.8). This claim follows from the monotonicity property. Let us consider the TPCP-map $\Phi_{A B}$ with two Kraus operators $\mathbb{1}_{A} \otimes \boldsymbol{\rho}_{B}^{0}$ and $\mathbb{1}_{A} \otimes\left(\mathbb{1}_{B}-\boldsymbol{\rho}_{B}^{0}\right)$. Due to (3.7), the state $\boldsymbol{\rho}_{A B}$ is not altered by the map $\Phi_{A B}$. For any sub-normalized state $\boldsymbol{\sigma}_{B} \in \mathcal{L}_{+}\left(\mathcal{H}_{B}\right)$, we further obtain

$$
\Phi_{A B}\left(\mathbb{1}_{A} \otimes \boldsymbol{\sigma}_{B}\right)=\mathbb{1}_{A} \otimes \boldsymbol{\sigma}_{B}^{\prime}, \quad \mu=\operatorname{tr}\left(\boldsymbol{\rho}_{B}^{0} \boldsymbol{\sigma}_{B}\right), \quad \bar{\mu}=\operatorname{tr}\left(\boldsymbol{\sigma}_{B}\right)-\mu .
$$

Using the relations (2.19) and (3.9), we immediately obtain the inequality

$$
D_{f}\left(\boldsymbol{\rho}_{A B} \| \mathbb{1}_{A} \otimes \mu \boldsymbol{\omega}_{B}\right) \leq D_{f}\left(\boldsymbol{\rho}_{A B} \| \mathbb{1}_{A} \otimes \boldsymbol{\sigma}_{B}\right),
$$

in which $\boldsymbol{\omega}_{B} \in \mathcal{L}_{+}\left(\operatorname{ran}\left(\boldsymbol{\rho}_{B}\right)\right)$ and $\operatorname{tr}\left(\boldsymbol{\omega}_{B}\right)=1$. Hence, the question is actually reduced to minimization over subnormalized states on the subspace $\operatorname{ran}\left(\boldsymbol{\rho}_{B}\right)$. Using item 2.3 of the paper [4], the left-hand side of (3.13) can be rewritten as

$$
\sum_{a \in \operatorname{spec}\left(\boldsymbol{\rho}_{A B}\right)}\left(\sum_{b \in \operatorname{spec}\left(\boldsymbol{\omega}_{B}\right) \backslash\{0\}} \mu b f\left(\frac{a}{\mu b}\right) \operatorname{tr}\left(\mathrm{P}_{a}\left(\mathbb{1}_{A} \otimes \boldsymbol{\pi}_{b}\right)\right)+a \ell(f) \operatorname{tr}\left(\mathrm{P}_{a}\left(\mathbb{1}_{A} \otimes \boldsymbol{\pi}_{0}\right)\right)\right) .
$$

Here, the projectors $\boldsymbol{\pi}_{b}$ correspond to the eigenvalues of $\boldsymbol{\omega}_{B}$. If the twice continuously differentiable function $\xi \mapsto f(\xi)$ is operator convex and $f(0)=0$, then the function $\xi \mapsto \xi^{-1} f(\xi)$ is operator monotone (see, e.g., Corollary V.3.11 of the book [39]). Hence, the quantity (3.14) does not increase with growth of $\mu$. To reach the infimum, we should set $\mu$ to its maximum, i.e., to $\mu=1$. Combining this with (3.5) gives the claim (3.11).

Under the conditions imposed on the function $\xi \mapsto f(\xi)$, the quantum conditional entropy (3.5) satisfies the properties (3.11) and (3.10). In other words, its value is independent of any extension of the used Hilbert space. The first condition is that the function should be operator convex. Then the $f$-divergence is monotone. We also demand that the function $\xi \mapsto \xi^{-1} f(\xi)$ be twice differentiable and $f(0)=0$. One of important examples is written as

$$
f_{\alpha}(\xi)=\frac{\xi^{\alpha}-\xi}{\alpha-1}
$$


including $f_{1}(\xi)=\xi \ln \xi$. The monotonicity property (2.19) is one of the key steps in the proof of Theorem 1 . The monotonicity of the $f$-divergence implies that important properties of the conditional entropy (3.5) hold. In the next section, we examine this question in more detail. For instance, we will show that the quantity (3.5) shares some properties similar to the results (3.2) and (3.3) for the standard conditional entropy (3.1). In this sense, the considered extension differs from a generalized conditional entropy determined by a local measurement [23]. The latter is always nonnegative and related to the quantum discord.

Let us discuss briefly computability of the quantum conditional entropy (3.5). The minimization of the $f$-divergence over $\boldsymbol{\sigma}_{B}$ in (3.11) is a nonlinear optimization problem with non-commuting variables. In general, such problems are sufficiently difficult. Even in the commutative case, various bounds on the $f$-divergences may be useful [25]. On the other hand, under some circumstances the quantum conditional entropy (3.5) is relatively easy to calculate or estimate analytically. For instance, more explicit relations can be obtained in the case of partly classical states. In more details, we will discuss results of such a kind in the next sections. Sometimes, an explicit form of the function $\xi \mapsto f(\xi)$ is required. As a significant particular example, we will consider the Tsallis case.

\section{SOME BASIC PROPERTIES}

In this section, we consider basic properties of quantum conditional entropies defined in terms of the $f$-divergences. In general, we are always interested in some bounds, which describe a range of possible values of studied quantities. Using arguments from the proof of Theorem 11 we can obtain an upper bound on the conditional entropy in terms of $\boldsymbol{\rho}_{A B}$. A simple lower bound easily follows from the definition of the conditional entropy. The following statement takes place.

Theorem 2 Let the function $\xi \mapsto f(\xi)$ satisfy all the preconditions of Theorem 1 , Then the quantum conditional entropy satisfies

$$
-d_{B}^{-1} \operatorname{tr}\left(f\left(d_{B} \boldsymbol{\rho}_{A B}\right)\right) \leq H_{f}\left(\boldsymbol{\rho}_{A B} \mid B\right) \leq-\operatorname{tr}\left(f\left(\boldsymbol{\rho}_{A B}\right)\right) .
$$

Proof. Let $\boldsymbol{\omega}_{B} \in \mathcal{L}_{+}\left(\mathcal{H}_{B}\right)$ be normalized state such that

$$
H_{f}\left(\boldsymbol{\rho}_{A B} \mid B\right)=-D_{f}\left(\boldsymbol{\rho}_{A B}|| \mathbb{1}_{A} \otimes \boldsymbol{\omega}_{B}\right) .
$$

The upper bound is based on non-decreasing of the function $\xi \mapsto \xi^{-1} f(\xi)$. Taking the expression (3.14) with $\mu=1$ and already without the second part, we get

$$
D_{f}\left(\boldsymbol{\rho}_{A B} \| \mathbb{1}_{A} \otimes \boldsymbol{\omega}_{B}\right) \geq D_{f}\left(\boldsymbol{\rho}_{A B} \| \mathbb{1}_{A} \otimes \mathbb{1}_{B}\right) .
$$

Indeed, eigenvalues of the normalized $\boldsymbol{\omega}_{B}$ do not exceed 1. It easily follows from (2.8) and (2.9) that

$$
D_{f}\left(\boldsymbol{\rho}_{A B} \| \mathbb{1}_{A} \otimes \mathbb{1}_{B}\right)=\operatorname{tr}\left(f\left(\boldsymbol{\rho}_{A B}\right)\right) .
$$

Combining the inequalities (4.3) and (4.4) with (4.2) finally gives the upper bound. Since $\boldsymbol{\omega}_{B}$ is optimizing density matrix, we further write

$$
D_{f}\left(\boldsymbol{\rho}_{A B} \| \mathbb{1}_{A} \otimes \boldsymbol{\omega}_{B}\right) \leq D_{f}\left(\boldsymbol{\rho}_{A B} \| \mathbb{1}_{A} \otimes \varrho_{* B}\right)
$$

where the completely mixed state $\varrho_{* B}=d_{B}^{-1} \mathbb{1}_{B}$. The operator $\mathbb{1}_{A} \otimes \varrho_{* B}$ has the eigenvalue $d_{B}^{-1}$ with multiplicity $d_{A} d_{B}$ and the projector $\mathbb{1}_{A} \otimes \mathbb{1}_{B}$. Reversing the sign in the right-hand side of (4.5), we merely reduce it to the left-hand side of (4.1).

Note that the proof of Theorem 2 tacitly uses (3.11), whence all the conditions on the function $\xi \mapsto f(\xi)$ are realized. In some interesting cases, the lower and upper bounds of Theorem 2 can be expressed in terms of a generalized entropy of state $\boldsymbol{\rho}_{A B}$. In the next section, we will consider an important case of the Tsallis entropies.

Under certain conditions of the used function, we will show the conditional entropy (3.5) to be strictly negative for pure entangled states. In this sense, the quantity (3.5) succeeds the relevant property (3.2) of the standard conditional entropy. Let twice continuously differentiable function $f(\xi)$ be operator convex and $f(0)=f(1)=0$. As was mentioned above, the function $\xi \mapsto \xi^{-1} f(\xi)$ is then non-decreasing. To estimate the infimum (3.11), we focus on finite-valued cases of the $f$-divergence. For a pure state $|A B\rangle \in \mathcal{H}_{A B}$, we write

$$
D_{f}\left(|A B\rangle\langle A B| \| \mathbb{1}_{A} \otimes \boldsymbol{\sigma}_{B}\right)=\sum_{b \in \operatorname{spec}\left(\boldsymbol{\sigma}_{B}\right) \backslash\{0\}} b f\left(\frac{1}{b}\right) \operatorname{tr}\left(\boldsymbol{\rho}_{B} \boldsymbol{\pi}_{b}\right) .
$$


Here, we used $f(0)=0$ and the term $\left\langle A B\left|\mathbb{1}_{A} \otimes \boldsymbol{\pi}_{b}\right| A B\right\rangle=\operatorname{tr}\left(\boldsymbol{\rho}_{B} \boldsymbol{\pi}_{b}\right)$, which follows from the Schmidt decomposition of $|A B\rangle$ and properties of the partial trace. In the considered case, we can rewrite (4.6) $\operatorname{merely}$ as $\operatorname{tr}\left(\boldsymbol{\rho}_{B} \boldsymbol{\sigma}_{B} f\left(\boldsymbol{\sigma}_{B}^{-1}\right)\right)$. Let us take $\boldsymbol{\sigma}_{B}$ as the completely mixed state on $\operatorname{ran}\left(\boldsymbol{\rho}_{B}\right)$, which reads $\operatorname{Sch}_{A B}^{-1} \boldsymbol{\rho}_{B}^{0}$ in terms of the Schmidt number $\operatorname{Sch}_{A B}=\operatorname{tr}\left(\boldsymbol{\rho}_{B}^{0}\right)$ of the state $|A B\rangle$. We then obtain from (4.6) a two-sided estimate

$$
0 \leq \inf _{\operatorname{tr}\left(\boldsymbol{\sigma}_{B}\right)=1} D_{f}\left(|A B\rangle\langle A B| \| \mathbb{1}_{A} \otimes \boldsymbol{\sigma}_{B}\right) \leq \frac{f\left(\operatorname{Sch}_{A B}\right)}{\operatorname{Sch}_{A B}} .
$$

Here, the zero bound follows as the function $b \mapsto b f(1 / b)$ is non-increasing, whence $b f(1 / b) \geq f(1)=0$ for $b \leq 1$. Then the quantum conditional entropy obeys

$$
-\frac{f\left(\operatorname{Sch}_{A B}\right)}{\operatorname{Sch}_{A B}} \leq H_{f}(|A B\rangle\langle A B|| B) \leq 0 .
$$

Thus, the quantum conditional entropy (3.5) of any bipartite pure state is not positive. We have arrived at this claim due to conditions that the used function is operator convex and $f(0)=f(1)=0$. We can improve the upper bound of (4.8), when the function $\xi \mapsto \xi^{-1} f(\xi)$ is additionally convex (for a concrete example, see the next section). Indeed, the function $b \mapsto b f(1 / b)$ then becomes also convex. Combining this with (4.6) further gives

$$
D_{f}\left(|A B\rangle\langle A B| \| \mathbb{1}_{A} \otimes \boldsymbol{\sigma}_{B}\right) \geq \operatorname{tr}\left(\boldsymbol{\rho}_{B} \boldsymbol{\sigma}_{B}\right) f\left(\operatorname{tr}\left(\boldsymbol{\rho}_{B} \boldsymbol{\sigma}_{B}\right)^{-1}\right) .
$$

Here, we used the Jensen inequality and the fact that probabilities $\operatorname{tr}\left(\boldsymbol{\rho}_{B} \boldsymbol{\pi}_{b}\right)$ are summarized to 1 . With $\operatorname{tr}\left(\boldsymbol{\sigma}_{B}\right)=1$, we clearly have $\operatorname{tr}\left(\boldsymbol{\rho}_{B} \boldsymbol{\sigma}_{B}\right) \leq\left\|\boldsymbol{\rho}_{B}\right\|_{\infty}$ (for a positive operator, its spectral norm is merely the maximum of eigenvalues). As the function $b \mapsto b f(1 / b)$ is non-increasing, the right-hand side of (4.9) is not less than $\left\|\boldsymbol{\rho}_{B}\right\|_{\infty} f\left(\left\|\boldsymbol{\rho}_{B}\right\|_{\infty}^{-1}\right)$. Hence, the quantum conditional entropy of a bipartite pure state is bounded from above as

$$
H_{f}\left(|A B\rangle\langle A B \| B) \leq-\left\|\boldsymbol{\rho}_{B}\right\|_{\infty} f\left(\left\|\boldsymbol{\rho}_{B}\right\|_{\infty}^{-1}\right)\right.
$$

We have $\left\|\boldsymbol{\rho}_{B}\right\|_{\infty} f\left(\left\|\boldsymbol{\rho}_{B}\right\|_{\infty}^{-1}\right)>f(1)=0$, when $\left\|\boldsymbol{\rho}_{B}\right\|_{\infty}<1$ and the function is not constant. The right-hand side of (4.10) and, therefore, the conditional entropy are strictly negative for pure entangled states. If Schmidt coefficients of the state $|A B\rangle$ are all equal, then $\boldsymbol{\rho}_{B}=\operatorname{Sch}_{A B}^{-1} \boldsymbol{\rho}_{B}^{0}$ and $\left\|\boldsymbol{\rho}_{B}\right\|_{\infty}=\operatorname{Sch}_{A B}^{-1}$. Then the right-hand side of (4.10) coincides with the left-hand side of (4.8). Thus, the lower bound of the relation (4.8) is saturated for such states, including maximally mixed states.

Thus, under certain conditions the presented generalization (3.5) succeeds one of the basic properties of the standard conditional entropy. In the next section, we will also exemplify that the quantity (3.5) obeys the property quite similar to (3.3). The following important property of quantum conditional entropies is related to data processing.

Theorem 3 Let $\Psi_{B}: \mathcal{L}\left(\mathcal{H}_{B}\right) \rightarrow \mathcal{L}\left(\mathcal{H}_{B^{\prime}}\right)$ be a TPCP-map, and let id ${ }_{A}$ be the identity map on $\mathcal{L}\left(\mathcal{H}_{A}\right)$. If the function $\xi \mapsto f(\xi)$ obeys all the preconditions of Theorem 1, then

$$
H_{f}\left(\boldsymbol{\rho}_{A B} \mid B\right) \leq H_{f}\left(\boldsymbol{\rho}_{A B^{\prime}} \mid B^{\prime}\right)
$$

where $\boldsymbol{\rho}_{A B^{\prime}}=\operatorname{id}_{A} \otimes \Psi_{B}\left(\boldsymbol{\rho}_{A B}\right)$.

Proof. Let $\boldsymbol{\omega}_{B} \in \mathcal{L}_{+}\left(\mathcal{H}_{B}\right)$ be normalized state such that the formula (4.2) holds. We first note that

$$
\operatorname{id}_{A} \otimes \Psi_{B}\left(\mathbb{1}_{A} \otimes \boldsymbol{\omega}_{B}\right)=\mathbb{1}_{A} \otimes \Psi_{B}\left(\boldsymbol{\omega}_{B}\right) .
$$

The monotonicity property (2.19) then gives

$$
D_{f}\left(\boldsymbol{\rho}_{A B^{\prime}} \| \mathbb{1}_{A} \otimes \Psi_{B}\left(\boldsymbol{\omega}_{B}\right)\right) \leq D_{f}\left(\boldsymbol{\rho}_{A B} \| \mathbb{1}_{A} \otimes \boldsymbol{\omega}_{B}\right) .
$$

Since the output $\Psi_{B}\left(\boldsymbol{\omega}_{B}\right)$ is a density matrix on $\mathcal{H}_{B^{\prime}}$, the definition (3.5) implies

$$
-D_{f}\left(\boldsymbol{\rho}_{A B^{\prime}}|| \mathbb{1}_{A} \otimes \Psi_{B}\left(\boldsymbol{\omega}_{B}\right)\right) \leq H_{f}\left(\boldsymbol{\rho}_{A B^{\prime}} \mid B^{\prime}\right) .
$$

Rearranging the two sides of (4.13) with the relevant sign, the formulas (4.2) and (4.14) provide the claim (4.11).

For the new version of conditional Rényi's entropy, an inequality of the form (4.11) has been obtained in [22]. We see that this inequality holds for an entire family of quantum conditional entropies based on the quantum $f$ divergences. An immediate corollary of the property (4.11) is posed as follows. Let $\boldsymbol{\rho}_{A B C}$ be density matrix on $\mathcal{H}_{A B C}=\mathcal{H}_{A} \otimes \mathcal{H}_{B} \otimes \mathcal{H}_{C}$. Then we have

$$
H_{f}\left(\boldsymbol{\rho}_{A B C} \mid B C\right) \leq H_{f}\left(\boldsymbol{\rho}_{A B} \mid B\right)
$$


where the density matrix $\boldsymbol{\rho}_{A B}$ is obtained from $\boldsymbol{\rho}_{A B C}$ by tracing-out $\mathcal{H}_{C}$. To check (4.15), we use the map id ${ }_{A} \otimes$ $\operatorname{id}_{B} \otimes \Psi_{C}$ with $\Psi_{C}$ defined for all $\left|c_{1}\right\rangle,\left|c_{2}\right\rangle \in \mathcal{H}_{C}$ as

$$
\Psi_{C}\left(\left|c_{2}\right\rangle\left\langle c_{1}\right|\right)=\left\langle c_{1} \mid c_{2}\right\rangle
$$

By linearity, the definition (4.16) is extended to all elements of $\mathcal{L}\left(\mathcal{H}_{C}\right)$. It is clear that the map $\Psi_{C}$ is a TPCP-map such that

$$
\operatorname{id}_{A} \otimes \operatorname{id}_{B} \otimes \Psi_{C}\left(\rho_{A B C}\right)=\rho_{A B}
$$

According to (4.15), conditioning on more can only reduce the entropy. It is of interest to obtain an inequality in opposite direction. Apparently, an explicit form of the used function $\xi \mapsto f(\xi)$ is required here. For the conditional Tsallis entropy, this issue will be considered below in Sect. V.

It is of interest to analyze properties of the quantum conditional entropy with respect to partly classical states. Let us take a collection of density matrices $\boldsymbol{\rho}_{A y} \in \mathcal{L}_{+}\left(\mathcal{H}_{A}\right)$ such that projectors $\boldsymbol{\rho}_{A y}^{0}$ are all mutually orthogonal, i.e.,

$$
\boldsymbol{\rho}_{A x}^{0} \perp \boldsymbol{\rho}_{A y}^{0} \quad(x \neq y),
$$

for all pairs $x \neq y$. To probability distribution $\left\{p_{y}\right\}$, we assign a density matrix

$$
\boldsymbol{\rho}_{A Y}=\sum_{y} p_{y} \boldsymbol{\rho}_{A y}
$$

It is said that the state (4.19) has a classical $Y$-register [22]. The following statement takes place.

Theorem 4 Let density matrix $\boldsymbol{\rho}_{A B Y} \in \mathcal{L}_{+}\left(\mathcal{H}_{A} \otimes \mathcal{H}_{B}\right)$ be given in the form

$$
\boldsymbol{\rho}_{A B Y}=\sum_{y} p_{y} \boldsymbol{\rho}_{A B y}
$$

in which $\boldsymbol{\rho}_{A B x}^{0} \perp \boldsymbol{\rho}_{A B y}^{0}$ for all $x \neq y$. Suppose also that $\boldsymbol{\rho}_{B x}^{0} \perp \boldsymbol{\rho}_{B y}^{0}$ for all $x \neq y$, where $\boldsymbol{\rho}_{B y}=\operatorname{tr}_{A}\left(\boldsymbol{\rho}_{A B y}\right)$ is the partial trace over $\mathcal{H}_{A}$. Let the function $\xi \mapsto f(\xi)$ satisfy all the preconditions of Theorem 1 , Then the quantum conditional entropy satisfies

$$
\sum_{y} p_{y} H_{f}\left(\boldsymbol{\rho}_{A B y} \mid B\right) \leq H_{f}\left(\boldsymbol{\rho}_{A B Y} \mid B Y\right)
$$

Proof. We recall already mentioned properties of the $f$-divergences. The first is expressed by the formula (3.6) $)$. For $\lambda \in[0 ;+\infty)$, we also use the homogeneity (2.17). By (3.7), for each $\boldsymbol{\rho}_{A B y}$ we have $\operatorname{ran}\left(\boldsymbol{\rho}_{A B y}\right) \subseteq \operatorname{ran}\left(\boldsymbol{\rho}_{A y}\right) \otimes \operatorname{ran}\left(\boldsymbol{\rho}_{B y}\right)$ in terms of the partial traces $\boldsymbol{\rho}_{A y}=\operatorname{tr}_{B}\left(\boldsymbol{\rho}_{A B y}\right)$ and $\boldsymbol{\rho}_{B y}=\operatorname{tr}_{A}\left(\boldsymbol{\rho}_{A B y}\right)$. So, we can treat $\boldsymbol{\rho}_{A B y}$ as a positive operator on $\mathcal{H}_{A} \otimes \operatorname{ran}\left(\boldsymbol{\rho}_{B y}\right)$. Let $\boldsymbol{\omega}_{B y} \in \mathcal{L}_{+}\left(\operatorname{ran}\left(\boldsymbol{\rho}_{B y}\right)\right)$ be density operator such that

$$
H_{f}\left(\boldsymbol{\rho}_{A B y} \mid B\right)=-D_{f}\left(\boldsymbol{\rho}_{A B y} \| \mathbb{1}_{A} \otimes \boldsymbol{\omega}_{B y}\right) .
$$

Introducing the state $\boldsymbol{\omega}_{B Y}=\sum_{y} p_{y} \boldsymbol{\omega}_{B y}$, we directly obtain

$$
\begin{aligned}
D_{f}\left(\boldsymbol{\rho}_{A B Y} \| \mathbb{1}_{A} \otimes \boldsymbol{\omega}_{B Y}\right) & =\sum_{y} D_{f}\left(p_{y} \boldsymbol{\rho}_{A B y} \| \mathbb{1}_{A} \otimes p_{y} \boldsymbol{\omega}_{B y}\right) \\
& =\sum_{y} p_{y} D_{f}\left(\boldsymbol{\rho}_{A B y} \| \mathbb{1}_{A} \otimes \boldsymbol{\omega}_{B y}\right)
\end{aligned}
$$

Since $\boldsymbol{\rho}_{B x}^{0} \perp \boldsymbol{\rho}_{B y}^{0}$ for $x \neq y$, the subspaces $\mathcal{H}_{A} \otimes \operatorname{ran}\left(\boldsymbol{\rho}_{B x}\right)$ and $\mathcal{H}_{A} \otimes \operatorname{ran}\left(\boldsymbol{\rho}_{B y}\right)$ contains mutually orthogonal vectors. Here, the step (4.23) is due to (3.6), and the step (4.24) is due to (2.17). According to (3.11), we also have

$$
-D_{f}\left(\boldsymbol{\rho}_{A B Y} \| \mathbb{1}_{A} \otimes \boldsymbol{\omega}_{B Y}\right) \leq H_{f}\left(\boldsymbol{\rho}_{A B Y} \mid B Y\right) .
$$

Rearranging the terms in (4.24) with the corresponding sign, we use (4.22) and (4.25) to complete the proof.

The result (4.21) relates the conditional entropy $H_{f}\left(\boldsymbol{\rho}_{A B Y} \mid B Y\right)$ with the conditional entropies of particular states $\boldsymbol{\rho}_{A B y}$. For the case of conditional Rényi entropies, a relation of such a kind has been derived in [22]. Moreover, the writers of [22] gave an exact relation. Below, we will obtain a similar exact relation for the Tsallis case. 


\section{QUANTUM CONDITIONAL ENTROPIES OF TSALLIS TYPE}

Let us consider the function (3.15) with positive $\alpha \neq 1$. In classical regime, the formula (2.1) leads to the Tsallis relative entropy

$$
S_{\alpha}(p \| q):=\frac{1}{1-\alpha}\left(1-\sum_{x} p_{x}^{\alpha} q_{x}^{1-\alpha}\right)=-\sum_{x} p_{x} \ln _{\alpha}\left(\frac{q_{x}}{p_{x}}\right) .
$$

This quantity was considered in [40]. In (5.1), the $\alpha$-logarithm is defined as

$$
\ln _{\alpha}(\xi):=\frac{\xi^{1-\alpha}-1}{1-\alpha}
$$

where $\alpha>0 \neq 1$ and $\xi>0$. As the function $f_{\alpha}(\xi)$ is convex and $f_{\alpha}(1)=0$, we have $S_{\alpha}(p \| q) \geq 0$ due to (2.2). In the limit $\alpha \rightarrow 1$, the $\alpha$-logarithm is reduced to the usual one. The quantity (5.1) then gives the standard relative entropy of probability distributions. Some bounds on the classical relative entropy (5.1) were obtained in [41, 42]. In quantum regime, this question has been addressed in [43].

Using (2.10) with the function $f_{\alpha}(\xi)$, we obtain the quantum version of Tsallis' relative entropy. For $\alpha \in(1 ;+\infty)$, the Tsallis $\alpha$-divergence is defined as

$$
D_{\alpha}(\mathrm{A} \| \mathrm{B}):= \begin{cases}\frac{1}{\alpha-1}\left(\operatorname{tr}\left(\mathrm{A}^{\alpha} \mathrm{B}^{1-\alpha}\right)-\operatorname{tr}(\mathrm{A})\right), & \text { if } \operatorname{ran}(\mathrm{A}) \subseteq \operatorname{ran}(\mathrm{B}), \\ +\infty, & \text { otherwise }\end{cases}
$$

For $\alpha \in(0 ; 1)$, the first entry of (5.3) is always suitable. According to (5.2), we have $f_{\alpha}(\xi)=\xi^{\alpha} \ln _{\alpha}(\xi)$. The standard relative entropy (2.11) is reached from (5.3) in the limit $\alpha \rightarrow 1$. The writers of 44 recently proposed a "sandwiched" relative Tsallis entropy connected with the new definition of Rényi's divergence [22, 26]. The corresponding conditional Tsallis form has been defined and used in identifying entanglement [44]. When the subsystem density matrix is a maximally mixed state, this "sandwiched" conditional Tsallis entropy gives the nonadditive conditional entropy proposed by Abe and Rajagopal [45]. Such an approach is beyond the scope of the present work.

The function $z \mapsto z^{\alpha}$ is operator concave on $[0 ;+\infty)$ for $0 \leq \alpha \leq 1$ and operator convex on $[0 ;+\infty)$ for $1 \leq \alpha \leq 2$ (see, e.g., items 4.2.3 and 1.5.8 in [47]). Hence, the function $f_{\alpha}(\xi)=\left(\xi^{\alpha}-\xi\right) /(\alpha-1)$ is operator convex for $\alpha \in(0 ; 2]$ and $\alpha \neq 1$. Combining this with the inequality (2.19) then gives

$$
D_{\alpha}(\Phi(\mathrm{A}) \| \Phi(\mathrm{B})) \leq D_{\alpha}(\mathrm{A} \| \mathrm{B}) .
$$

For $\alpha \in(0 ; 2]$, the quantum Tsallis divergence is monotone under the action of trace-preserving completely positive maps. Monotonicity of the divergence leads to a lot of properties of the conditional Tsallis entropy. Since $f_{\alpha}(0)=0$, we apply (3.11) and obtain the quantum conditional entropy

$$
H_{\alpha}\left(\boldsymbol{\rho}_{A B} \mid B\right):=-\inf \left\{D_{\alpha}\left(\boldsymbol{\rho}_{A B}|| \mathbb{1}_{A} \otimes \boldsymbol{\sigma}_{B}\right): \boldsymbol{\sigma}_{B} \in \mathcal{L}_{+}\left(\mathcal{H}_{B}\right), \operatorname{tr}\left(\boldsymbol{\sigma}_{B}\right)=1\right\} .
$$

In this definition, we will assume $\alpha \in(0 ; 2]$, whence the Tsallis $\alpha$-divergence (5.3) is monotone.

Let us discuss briefly the conditional Tsallis entropy of pure states. We first note that the function

$$
\xi^{-1} f_{\alpha}(\xi)=\frac{\xi^{\alpha-1}-1}{\alpha-1}=-\ln _{\alpha}\left(\frac{1}{\xi}\right)
$$

is increasing and convex for $\alpha \in(0 ; 2]$. Combining this with (4.8) and (4.10) gives a two-sided estimate

$$
\ln _{\alpha}\left(\frac{1}{\operatorname{Sch}_{A B}}\right) \leq H_{\alpha}\left(|A B\rangle\langle A B|| B) \leq \ln _{\alpha}\left(\left\|\boldsymbol{\rho}_{B}\right\|_{\infty}\right)\right.
$$

For entangled pure states, we have $\left\|\boldsymbol{\rho}_{B}\right\|_{\infty}<1$ and strictly negative value of the conditional Tsallis entropy. The lower and upper bounds of the relation (5.7) coincide in the case, when Schmidt coefficients of the state $|A B\rangle$ are all equal. Then the conditional Tsallis entropy is equal to $\ln _{\alpha}\left(\operatorname{Sch}_{A B}^{-1}\right)$, including $-\ln \left(\operatorname{Sch}_{A B}\right)$ for the usual case $\alpha=1$. We further consider the conditional Tsallis entropy of separable states. Using the representation (5.3), for any $\boldsymbol{\sigma}_{B} \in \mathcal{L}_{+}\left(\operatorname{ran}\left(\boldsymbol{\rho}_{B}\right)\right)$ with the same range we write

$$
\begin{aligned}
D_{\alpha}\left(\boldsymbol{\rho}_{A} \otimes \boldsymbol{\rho}_{B} \| \mathbb{1}_{A} \otimes \boldsymbol{\sigma}_{B}\right) & =\frac{1}{\alpha-1}\left(\operatorname{tr}\left(\boldsymbol{\rho}_{A}^{\alpha}\right) \operatorname{tr}\left(\boldsymbol{\rho}_{B}^{\alpha} \boldsymbol{\sigma}_{B}^{1-\alpha}\right)-1\right) \\
& =\operatorname{tr}\left(\boldsymbol{\rho}_{A}^{\alpha}\right) D_{\alpha}\left(\boldsymbol{\rho}_{B} \| \boldsymbol{\sigma}_{B}\right)-H_{\alpha}\left(\boldsymbol{\rho}_{A}\right)
\end{aligned}
$$


Here, the quantum Tsallis $\alpha$-entropy of normalized state $\boldsymbol{\rho}_{A}$ is defined as

$$
H_{\alpha}\left(\boldsymbol{\rho}_{A}\right):=\frac{1}{\alpha-1}\left(\operatorname{tr}\left(\boldsymbol{\rho}_{A}^{\alpha}\right)-1\right)=-D_{\alpha}\left(\boldsymbol{\rho}_{A} \| \mathbb{1}_{A}\right) .
$$

Properties of the quantum Tsallis entropy are discussed in [46]. With normalized $\boldsymbol{\sigma}_{B}$, we have $D_{\alpha}\left(\boldsymbol{\rho}_{B} \| \boldsymbol{\sigma}_{B}\right) \geq 0$ (see (2.16) and related comments). That is, the infimum of (5.8) is equal to $-H_{\alpha}\left(\boldsymbol{\rho}_{A}\right)$; it is clearly reached for $\boldsymbol{\sigma}_{B}=\boldsymbol{\rho}_{B}$. From (5.5), we finally obtain

$$
H_{\alpha}\left(\boldsymbol{\rho}_{A} \otimes \boldsymbol{\rho}_{B} \mid B\right)=H_{\alpha}\left(\boldsymbol{\rho}_{A}\right) .
$$

Thus, for $\alpha \in(0 ; 2]$ the quantum conditional entropy (5.5) succeeds the property (3.3) of the standard conditional entropy (3.1). It is certainly nonnegative in the case of separable mixed states.

Applying (4.1) with $f_{\alpha}(\xi)=\left(\xi^{\alpha}-\xi\right) /(\alpha-1)$, we obtain the corresponding lower and upper bounds:

$$
d_{B}^{\alpha-1} H_{\alpha}\left(\boldsymbol{\rho}_{A B}\right)+\ln _{\alpha}\left(d_{B}^{-1}\right) \leq H_{\alpha}\left(\boldsymbol{\rho}_{A B} \mid B\right) \leq H_{\alpha}\left(\boldsymbol{\rho}_{A B}\right) .
$$

These bounds are based on (3.11) and hold for all $\alpha \in(0 ; 2]$. In the standard case $\alpha=1$, these bounds are merely reduced to

$$
H_{1}\left(\boldsymbol{\rho}_{A B}\right)-\ln d_{B} \leq H_{1}\left(\boldsymbol{\rho}_{A B} \mid B\right) \leq H_{1}\left(\boldsymbol{\rho}_{A B}\right) .
$$

Of course, this claim also follows from (3.1) due to $0 \leq H_{1}\left(\boldsymbol{\rho}_{B}\right) \leq \ln d_{B}$. The result (5.11) is an extension of (5.12) to conditional Tsallis' entropies.

The results (4.11) and (4.15) are based on the monotonicity property. They hold for the conditional Tsallis $\alpha$-entropy of degree $\alpha \in(0 ; 2]$. In particular, we have

$$
H_{\alpha}\left(\boldsymbol{\rho}_{A B C} \mid B C\right) \leq H_{\alpha}\left(\boldsymbol{\rho}_{A B} \mid B\right) .
$$

As was already mentioned, we can obtain an inequality in opposite direction. Namely, we have the following statement.

Theorem 5 Let $\boldsymbol{\rho}_{A B C}$ be density matrix on the product $\mathcal{H}_{A B C}=\mathcal{H}_{A} \otimes \mathcal{H}_{B} \otimes \mathcal{H}_{C}$. For all $\alpha \in(0 ; 2]$, the quantum conditional $\alpha$-entropy satisfies

$$
H_{\alpha}\left(\boldsymbol{\rho}_{A B C} \mid B\right) \leq d_{C}^{1-\alpha} H_{\alpha}\left(\boldsymbol{\rho}_{A B C} \mid B C\right)+\ln _{\alpha}\left(d_{C}\right),
$$

where $d_{C}$ is the dimensionality of $\mathcal{H}_{C}$.

Proof. We also suppose $\alpha \neq 1$. Let $\boldsymbol{\omega}_{B} \in \mathcal{L}_{+}\left(\mathcal{H}_{B}\right)$ be normalized state such that

$$
H_{\alpha}\left(\boldsymbol{\rho}_{A B C} \mid B\right)=-D_{\alpha}\left(\boldsymbol{\rho}_{A B} \| \mathbb{1}_{A} \otimes \boldsymbol{\omega}_{B} \otimes \mathbb{1}_{C}\right) .
$$

Using the completely mixed state $\boldsymbol{\varrho}_{* C}=d_{C}^{-1} \mathbb{1}_{C}$ on $\mathcal{H}_{C}$, we write

$$
\begin{aligned}
D_{\alpha}\left(\boldsymbol{\rho}_{A B} \| \mathbb{1}_{A} \otimes \boldsymbol{\omega}_{B} \otimes \mathbb{1}_{C}\right) & =\frac{1}{\alpha-1}\left[d_{C}^{1-\alpha} \operatorname{tr}\left(\boldsymbol{\rho}_{A B C}^{\alpha}\left(\mathbb{1}_{A} \otimes \boldsymbol{\omega}_{B} \otimes \boldsymbol{\varrho}_{* C}\right)^{1-\alpha}\right)-1\right] \\
& =d_{C}^{1-\alpha} D_{\alpha}\left(\boldsymbol{\rho}_{A B} \| \mathbb{1}_{A} \otimes \boldsymbol{\omega}_{B} \otimes \boldsymbol{\varrho}_{* C}\right)-\ln _{\alpha}\left(d_{C}\right) .
\end{aligned}
$$

According to the definition (5.5), we also have

$$
-D_{\alpha}\left(\boldsymbol{\rho}_{A B}|| \mathbb{1}_{A} \otimes \boldsymbol{\omega}_{B} \otimes \boldsymbol{\varrho}_{* C}\right) \leq H_{\alpha}\left(\boldsymbol{\rho}_{A B C} \mid B C\right),
$$

since the product $\boldsymbol{\omega}_{B} \otimes \varrho_{* C}$ is a density matrix on $\mathcal{H}_{B C}=\mathcal{H}_{B} \otimes \mathcal{H}_{C}$. After substituting (5.16) into (5.15), the relation (5.17) completes the proof for $\alpha \neq 1$. The case $\alpha=1$ can be resolved in a similar manner.

Analogous property has been proved for the conditional min-entropy by Renner [20] and for the new form of conditional Rényi's entropy in [22]. This property is usually referred to as the chain rule. Thus, we have derived the chain rule (5.14) in the Tsallis formulation. Note that the proof of Theorem 5 tacitly uses the result (3.11), whence the restriction $\alpha \in(0 ; 2]$ occurs.

In conclusion, we consider the conditional Tsallis entropy of partly classical states. The inequality (4.21) has been derived for any function $\xi \mapsto f(\xi)$ that obeys the preconditions of Theorem 1. When this function is given explicitly, we could obtain an exact relation instead of (4.21). For the Tsallis case, it is posed as follows. 
Theorem 6 Let density matrix $\boldsymbol{\rho}_{A B Y} \in \mathcal{L}_{+}\left(\mathcal{H}_{A} \otimes \mathcal{H}_{B}\right)$ be given by 4.20), and let projectors $\boldsymbol{\rho}_{A B y}^{0}$ and $\boldsymbol{\rho}_{B y}^{0}$ obey the same preconditions as in Theorem 母. For $\alpha \in(0 ; 2]$, the conditional Tsallis entropy satisfies

$$
H_{\alpha}\left(\boldsymbol{\rho}_{A B Y} \mid B Y\right)=\frac{1}{1-\alpha}\left[\left(\sum_{y} p_{y}\left(1+(1-\alpha) H_{\alpha}\left(\boldsymbol{\rho}_{A B y} \mid B\right)\right)^{1 / \alpha}\right)^{\alpha}-1\right] .
$$

Proof. We first assume that $\alpha \neq 1$. According to the definition (5.5), we aim to find the infimum of $D_{\alpha}\left(\boldsymbol{\rho}_{A B Y} \| \mathbb{1}_{A} \otimes \boldsymbol{\sigma}_{B Y}\right)$ over all density matrices $\boldsymbol{\sigma}_{B Y}$ on $\mathcal{H}_{B}$. Following [22], we first note that the optimization can be taken over states of the form

$$
\boldsymbol{\sigma}_{B Y}^{\prime}=\sum_{y} q_{y} \boldsymbol{\sigma}_{B y}
$$

Here, the set $\left\{q_{y}\right\}$ is some probability distribution and $\boldsymbol{\sigma}_{B y} \in \mathcal{L}_{+}\left(\operatorname{ran}\left(\boldsymbol{\rho}_{B y}\right)\right)$. The reason is posed as follows. Adding zero probabilities to $\left\{p_{y}\right\}$, we can always assume the set of projectors $\boldsymbol{\rho}_{B y}^{0}$ to be complete in $\mathcal{H}_{B}$, namely

$$
\sum_{y} \rho_{B y}^{0}=\mathbb{1}_{B}
$$

We now apply a TPCP-map $\Phi_{A B Y}$ with Kraus operators $\mathrm{K}_{y}=\mathbb{1}_{A} \otimes \boldsymbol{\rho}_{B y}^{0}$. Due to $\boldsymbol{\rho}_{B x}^{0} \perp \boldsymbol{\rho}_{B y}^{0}$ for $x \neq y$, this map transforms an entry $\mathbb{1}_{A} \otimes \boldsymbol{\sigma}_{B Y}$ with any $\boldsymbol{\sigma}_{B Y}$ into an entry $\mathbb{1}_{A} \otimes \boldsymbol{\sigma}_{B Y}^{\prime}$ with a density matrix $\boldsymbol{\sigma}_{B Y}^{\prime}$ of the form (5.19). The state (4.20) remains unaltered, since $\operatorname{ran}\left(\boldsymbol{\rho}_{A B y}\right) \subseteq \mathcal{H}_{A} \otimes \operatorname{ran}\left(\boldsymbol{\rho}_{B y}\right)$. By the monotonicity (5.4), the divergence cannot increase under the action of the map $\Phi_{A B Y}$, as claimed. For the states (4.20) and (5.19), immediate calculations give

$$
D_{\alpha}\left(\boldsymbol{\rho}_{A B Y} \| \mathbb{1}_{A} \otimes \boldsymbol{\sigma}_{B Y}^{\prime}\right)=S_{\alpha}(p \| q)+\sum_{y} p_{y}^{\alpha} q_{y}^{1-\alpha} D_{\alpha}\left(\boldsymbol{\rho}_{A B y} \| \mathbb{1}_{A} \otimes \boldsymbol{\sigma}_{B y}\right),
$$

where we used (3.6) (5.1), and (5.3). The right-hand side of (5.21) should be minimized over all probability distributions $\left\{q_{y}\right\}$ and state collections $\left\{\boldsymbol{\sigma}_{B y}\right\}$. Let $\boldsymbol{\omega}_{B} \in \mathcal{L}_{+}\left(\mathcal{H}_{B}\right)$ be density operator such that the equality (4.22) actually holds. The minimization over $\left\{\boldsymbol{\sigma}_{B y}\right\}$ merely leads to substituting the collection $\left\{\boldsymbol{\omega}_{B y}\right\}$. Indeed, the factor of each $\alpha$-divergence in the right-hand side of (5.21) is nonnegative. Then we have

$$
-H_{\alpha}\left(\boldsymbol{\rho}_{A B Y} \mid B Y\right)=\inf \left\{\frac{1}{\alpha-1}\left(\sum_{y} p_{y}^{\alpha} q_{y}^{1-\alpha} t_{y}-1\right): q_{y} \geq 0, \sum_{y} q_{y}=1\right\}
$$

where we fix the distribution $\left\{p_{y}\right\}$ and auxiliary parameters

$$
t_{y}=1+(1-\alpha) H_{\alpha}\left(\boldsymbol{\rho}_{A B y} \mid B\right)=\operatorname{tr}\left(\boldsymbol{\rho}_{A B y}^{\alpha}\left(\mathbb{1}_{A} \otimes \boldsymbol{\omega}_{B y}\right)^{1-\alpha}\right) .
$$

Clearly, these parameters are nonnegative. In the term (15.22), therefore, we deal with a convex function of the variables $q_{y}$. Indeed, its second derivative with respect to $q_{x}$ reads $\alpha p_{x}^{\alpha} t_{x} q_{x}^{-\alpha-1}$ and is nonnegative for $\alpha>0$. The method of Lagrange multipliers is a standard tool to fit problems of minimizing convex function subject to convex constraints [48]. Applying this technique to the task (5.22) leads to the answer

$$
q_{x}=\left(\sum_{y} t_{y}^{1 / \alpha} p_{y}\right)^{-1} t_{x}^{1 / \alpha} p_{x}
$$

which takes into account the normalization condition. Substituting the formulas (5.23) and (5.24) and into the righthand side of (5.22) finally gives (5.18). By inspection of second derivatives, we see that the found point is a conditional minimum. The proof in the standard case $\alpha=1$ is short. Using (2.11), we immediately obtain the formula

$$
D_{1}\left(\boldsymbol{\rho}_{A B Y} \| \mathbb{1}_{A} \otimes \boldsymbol{\sigma}_{B Y}^{\prime}\right)=S_{1}(p \| q)+\sum_{y} p_{y} D_{1}\left(\boldsymbol{\rho}_{A B y} \| \mathbb{1}_{A} \otimes \boldsymbol{\sigma}_{B y}\right),
$$

which is also a limit case of the result (5.21). Two summands in the right-hand side of (5.25) are minimized independently. Substituting the collection $\left\{\boldsymbol{\omega}_{B y}\right\}$ optimizes the second summand over $\left\{\boldsymbol{\sigma}_{B y}\right\}$. The first summand obeys $S_{1}(p \| q) \geq 0$ (see, e.g., theorem 11.1 in [19]) and vanishes, when $q_{y}=p_{y}$ for all $y$. Hence, we obtain the result

$$
H_{1}\left(\boldsymbol{\rho}_{A B Y} \mid B Y\right)=\sum_{y} p_{y} H_{1}\left(\boldsymbol{\rho}_{A B y} \mid B\right)
$$

which follows from (5.18) in the limit $\alpha \rightarrow 1$. 
The statement of Theorem 6 gives an expression of the conditional Tsallis $\alpha$-entropy in the case of partly classical states. For the conditional Rényi entropy, this issue was already considered in [22]. Note that the expression (5.26]) for $\alpha \rightarrow 1$ follows from the Rényi formulation as well. As a particular case of (5.18), we obtain the result

$$
H_{\alpha}\left(\boldsymbol{\rho}_{A Y} \mid Y\right)=\frac{1}{1-\alpha}\left[\left(\sum_{y} p_{y}\left(1+(1-\alpha) H_{\alpha}\left(\boldsymbol{\rho}_{A y}\right)\right)^{1 / \alpha}\right)^{\alpha}-1\right]
$$

in which conditioning is purely classical. Here, the restriction $\alpha \in(0 ; 2]$ is essential again. We see that the conditional Tsallis entropy obeys some relations similarly to the conditional Rényi entropy.

\section{CONCLUSIONS}

We have studied quantum conditional entropies defined in terms of the $f$-divergences. This approach has previously been applied to the so-called min-entropy and max-entropy in [20, 21]. Recently, it was considered for the Rényi case [22]. We have applied the mentioned method to a family of the quantum $f$-divergences. We gave explicit conditions, when the notion of quantum conditional entropies is well defined in the developed approach. This question is naturally raised from the fact that any quantum system can always be imagined as a part of larger quantum systems. The additional conditions on $f$ are that it is twice continuously differentiable and $f(0)=0$. Together with the operator convexity, the conditions imply non-decreasing of the function $\xi \mapsto \xi^{-1} f(\xi)$. When other conditions are already satisfied, this property can easily be reached.

Assuming these conditions, important properties of the introduced conditional entropies have been discussed. In particular, the presented quantity resembles properties of the standard conditional entropy in both the cases of pure states and separable states. We also derived simple lower and upper bounds on the conditional entropy in terms of the corresponding density matrix. A behavior with respect to data processing was considered. Quantum conditional entropies of partly classical states were examined. We have considered an especially important case of the quantum conditional entropies of Tsallis type. In some respects, their properties are similar to quantum conditional Rényi entropies recently studied in [22]. The presented discussion is a further development of the approach originally proposed in [20, 21].

[1] V. Vedral: Rev. Mod. Phys. 74, 197-234 (2002).

[2] D. Petz: Quantum Information Theory and Quantum Statistics, Springer, Berlin 2008.

[3] I. Bengtsson and K. Życzkowski: Geometry of Quantum States: An Introduction to Quantum Entanglement, Cambridge University Press, Cambridge 2006.

[4] F. Hiai, M. Mosonyi, D. Petz and C. Bény: Rev. Math. Phys. 23, 691-747 (2011).

[5] I. Csiszár: Studia Sci. Math. Hungar. 2, 299-318 (1967).

[6] D. Petz: Rep. Math. Phys. 21, 57-65 (1986).

[7] K. M. R. Audenaert: Quantum. Inf. Comput. 14, 0031-0038 (2013).

[8] N. Ciganović, N. J. Beaudry, and R. Renner: Smooth max-information as one-shot generalization for mutual information, arXiv:1308.5884 [math-ph] (2013).

[9] T. M. Cover and J. A. Thomas: Elements of Information Theory, John Wiley \& Sons, New York 1991.

[10] D. Erdogmus and J. C. Principe: J. VLSI Signal Process. 37, 305-317 (2004).

[11] S. Furuichi: J. Math. Phys. 47, 023302 (2006).

[12] A. Rényi: On Measures of Entropy and Information, in Proc. 4th Berkeley Symp. on Mathematical Statistics and Probability, J. Neyman ed., 547-561, University of California Press, Berkeley, CA 1961.

[13] C. Tsallis: J. Stat. Phys. 52, 479-487 (1988).

[14] A. E. Rastegin: Kybernetika 48, 242-253 (2012).

[15] L. B. Levitin: Chaos, Solitons \& Fractals 10, 1651-1656 (1999).

[16] R. Schrader: Fortschr. Phys. 48, 747-762 (2000).

[17] E. H. Lieb: Bull. Am. Math. Soc.. 81, 1-13 (1975).

[18] E. H. Lieb and M. B. Ruskai: J. Math. Phys. 14, 1938-1941 (1973).

[19] M. A. Nielsen and I. L. Chuang: Quantum Computation and Quantum Information, Cambridge University Press, Cambridge 2000.

[20] R. Renner: Security of quantum key distribution, PhD thesis, ETH Zurich, arXiv: quant-ph/0512258 (2005).

[21] R. König, R. Renner and C. Schaffner: IEEE Trans. Inf. Theory 55, 4337-4347 (2009).

[22] M. Müller-Lennert, F. Dupuis, O. Szehr, S. Fehr and M. Tomamichel: J. Math. Phys. 54, 122203 (2013).

[23] N. Gigena and R. Rossignoli: J. Phys. A: Math. Theor. 47, 015302 (2014).

[24] I. Csiszár: Entropy 10, 261-273 (2008). 
[25] S. S. Dragomir: Appl. Math. 48, 205-223 (2003).

[26] M. M. Wilde, A. Winter, and D. Yang: Strong converse for the classical capacity of entanglement-breaking and Hadamard channels, arXiv:1306.1586] [quant-ph] (2013).

[27] K. M. R. Audenaert and N. Datta: $\alpha$-z-relative Rényi entropies, arXiv:1310.7178 [quant-ph] (2013).

[28] M. M. Wilde and A. Winter: Strong converse for the classical capacity of the pure-loss bosonic channel, arXiv:1308.6732 [quant-ph] (2013).

[29] R. L. Frank and E. H. Lieb: J. Math. Phys. 54, 122201 (2013).

[30] S. Beigi: J. Math. Phys. 54, 122202 (2013).

[31] M. Mosonyi and T. Ogawa: Quantum hypothesis testing and the operational interpretation of the quantum Rényi relative entropies, arXiv:1309.3228 [quant-ph] (2013).

[32] W. Roga, M. Fannes and K. Życzkowski: Phys. Rev. Lett. 105, 040505 (2010).

[33] W. Roga, M. Fannes, and K. Życzkowski: Int. J. Quantum Inf. 9, 1031-1046 (2011).

[34] A. E. Rastegin: J. Phys. A: Math. Theor. 45, 045302 (2012).

[35] A. E. Rastegin: Cent. Eur. J. Phys. 11, 69-77 (2013).

[36] A. E. Rastegin: J. Stat. Phys. 148, 1040-1053 (2012).

[37] R. Alicki and M. Fannes: J. Phys. A: Math. Gen. 37, L55-L57 (2004).

[38] M. Tomamichel: A framework for non-asymptotic quantum information theory, PhD thesis, ETH Zurich, arXiv:1203.2142 [quant-ph] (2012).

[39] R. Bhatia: Matrix Analysis, Springer, New York 1997.

[40] L. Borland, A. R. Plastino, and C. Tsallis: J. Math. Phys. 39, 6490-6501 (1998).

[41] I. Csiszár: Studia Sci. Math. Hungar. 1, 227-230 (1966).

[42] G. Gilardoni: IEEE Trans. Inf. Theory 56, 5377-5386 (2010).

[43] A. E. Rastegin: Math. Phys. Anal. Geom. 16, 213-228 (2013).

[44] A. K. Rajagopal, Sudha, A. S. Nayak, and A. R. Usha Devi: Phys. Rev. A 89, 012331 (2014).

[45] S. Abe and A. K. Rajagopal: Physica A 289, 157-164 (2001).

[46] G. A. Raggio: J. Math. Phys. 36, 4785-4791 (1995).

[47] R. Bhatia: Positive Definite Matrices, Princeton University Press, Princeton 2007.

[48] R. T. Rockafellar: Convex Analysis, Princeton University Press, Princeton 1970. 